\title{
Some Aspects of the Modern Development of Magnetic Resonance
}

\author{
Kev M. Salikhov
}

Published online: 23 July 2013

(C) Springer-Verlag Wien 2013

Methods of magnetic resonance including those of electron paramagnetic/spin resonance (EPR/ESR) are widely used in diverse fields of science and medicine, and they contribute a lot to the development of the new (spin) technologies, etc.

Spin resonance methodologies and their promising applications were first started and developed in Russia, USA, United Kingdom, Germany, France and other countries. At present the magnetic resonance methods are successfully used all over the world. This issue of Applied Magnetic Resonance is a vivid indication of the inspiring development of the EPR/ESR methodology by the researchers in the AsiaPacific countries.

It is my pleasure to present the authors of this issue and to comment in short about the content of the papers. The geography of the affiliation of their authors is quite impressive.

Guo Junwang, Cong Jianbo and Wu Ke developed a specially designed cavity for performing in vivo ESR dose measurements in X-band. Their paper introduces the operation principle, configuration, and the theoretical calculation of the cavity and experiments on the newly developed X-band ESR cavity. The preliminary results of experiments are also presented and discussed. N.V. Ivanisenko and S.A. Dzuba have shown the usefulness of spin-echo EPR of spin labels when applied to investigate the molecular mechanisms of action of cryoprotective agents on biological systems. Kohdai Fujimoto, Takahiro Sakurai, Susumu Okubo, Hitoshi Ohta, Kazuyuki Matsubayashi, Yoshiya Uwatoko, Kazutaka Kudo and Yoji Koike have developed a hybrid-type piston-cylinder pressure cell and a high-field ESR system using a cryogen-free superconducting magnet for this pressure cell. This is expected to be a promising tool to study novel pressure-induced phenomena. C. Rudowicz, P. Gnutek, S. Kimura, M. Açıkgöz and Y. Y. Yeung are developing new approaches to

K. M. Salikhov $(\bowtie)$

Zavoisky Physical-Technical Institute, Russian Academy of Sciences,

Kazan 420029, Russian Federation

e-mail: salikhov@kfti.knc.ru 
modeling of the spin-Hamiltonian parameters. Lin-Hua Xie and Yau-Yuen Yeung have investigated impurity structures using EPR spectroscopy and molecular dynamics calculations, and demonstrated that these two approaches provide complementary information. Simon C. Drew, W. Mei Kok, Craig A. Hutton and Kevin J. Barnham attempt to elucidate the molecular mechanism of toxicity of lowmolecular-weight amyloid- $\beta$ peptide oligomers in Alzheimer's disease. To investigate structural aspects of psoriasis vulgaris SC, Kouichi Nakagawa, Satoko Minakawa and Daisuke Sawamura have used the EPR spin-probe method and analyzed the order parameter and the molecular rotational diffusion coefficient. K. B. Konov, A. A. Knyazev, Yu. G. Galyametdinov, N. P. Isaev and L. V. Kulik have introduced a dead-time free version of relaxation-induced dipolar modulation enhancement experiment. This method was tested on a rigid nitroxide biradical in frozen solution. Yu E. Kandrashkin, V. S. Iyudin, V. K. Voronkova, E. A. Mikhalitsyna and V. S. Tyurin present EPR evidence of the dimerization of the aza-crown copper porphyrins. The EPR data manifest the chemically induced dynamic electron polarization effect for the excited state of the supramolecule formed by the interaction of the photoexcited porphyrin in the quartet state and the nearby non-excited porphyrin in the ground state. Jörg Reichenwallner, Mohammed Chakour, S. Indu, Raghavan Varadarajan and Wolfgang E. Trommer have presented interesting results concerning structures of cysteines obtained by combining the spin labeling of proteins and double electron-electron pulse EPR method.

This issue of Applied Magnetic Resonance was edited by Professors Sergei A. Dzuba (Russia), Gareth R. Eaton (USA), Sandra S. Eaton (USA) and Yong Li (China). They did a great job and I am most appreciative of their time and efforts.

Kev M. Salikhov

Editor-in-chief 